\title{
Prevalence of Postpartum Distress and Accessibility to Mental Health Services in South Darfur, Nyala: April- May, 2018
}

\author{
Ekram AE ${ }^{*}$, Babikir $\mathrm{AN}^{2}$ and Hawa $\mathrm{ZI}^{3}$ \\ 1Faculty of Public Health, University of Alzaeim Alazhari, Sudan \\ ${ }^{2}$ Health Officer Unicef, South Darfur, Sudan \\ ${ }^{3}$ American Refugee Committee, South Darfur Nyala, Sudan
}

\section{Research Article \\ Volume 2 Issue 2}

Received Date: July 17, 2018

Published Date: September 06, 2018

DOI: $10.23880 /$ phoa- 16000130

*Corresponding author: Ekram Adam Eldooma, Faculty of Public Health, University of Alzaeim Alazhari, Khartoum North, Sudan, Email: ekram.eldoom3@gmail.com

\section{Abstract}

Introduction: Post-partum mental distress is recently recognized type of mental disease that can badly affect women after delivery in case the condition is not well addressed and treated promptly.

Objectives: To estimate the prevalence of post-partum distress and the accessibility to mental health services in South Darfur-Nyala City.

Methodology: Convenience sampling was done 60 women in post-natal ward. Two questionnaires have been filled to assess the both prevalence of post-partum distress and the accessibility to mental services in Nyala city.

Results: 35 women in the age group (21-25years) at 58.4\% are predominant out of 60 women in developing mental health services. 51 women (85\%) out of the total are house wives with great implication of daily tasks resulting in postnatal distress. $75.6 \%$ are normal delivery while $24.4 \%$ have been delivered by caesarian section.

Recommendation: Awareness is the key element in improving the overall accessibility to mental health services and reducing the prevalence of the post-partum distress in women in this period. This is could be done through mental health campaigns.

Conclusion: Competing factors have been the root cause in women presenting with mental distress during the postpartum period of which the overburden on women in taking care of expanded families, the poor education and the low income for most of families which as well a major factor in developing post-partum distress.

Keywords: Delivery; Postnatal Depression; Psychological; Partner Support; Socio-Demographic Factors; Psycho-Social Factors

\section{Background}

WHO defines Health as the state of complete physical, mental and social wellbeing and not merely absence of disease or infirmity? [1]
Despite the mental disorders that predominately occur in general population in the recent years which have been shown in couples of studies and contributing mainly to different factors, still lack of data and studies linking postpartum period and the mental distress is an 
area of concern and requires more research and explore [2].

Postpartum depression is one of the most complications characterized by behavioral problems and mental disorders in women about 4-6 weeks after giving birth. However, there is big variation in prevalence from one country to another. The prevalence has shown 10 $15 \%$ of mothers in western economically developed societies [3]. Iran has shown prevalence at $34 \%$ while another study in Korea has resulted in $36.7 \%$ at 6 weeks after delivery. This variation in prevalence rates could be attributable to distinct time of study, the assessment tools used, the diagnostic criteria, biologic vulnerability and difference in cultural and socioeconomic environment [4].

More than $85 \%$ of world population lives in 153 low and middle incomes countries. Most of these countries allocate scarce financial resources and limited manpower and have grossly inadequate manpower and infrastructure for mental health. Many of low and middle income countries lack mental health policy and legislation to direct their mental health program and services.

Factors for paternal PPD included negative emotions, financial concerns, balancing work-life demands, low education levels, and marital problems. In addition to depression, other mental health issues occurred for some fathers in the postpartum period, including helplessness and anxiety [5].

The prevalence has steadily increase from $8 \%$ in 1950 to reach $27 \%$ in 2015 [6].This increment could not be attributed to one cause with many factors being incriminated directly or indirectly leading to this prevalence with big portion claimed in the stress life style these days and minor support that could be available to individuals suffering from different kind of mental disease and in particular women in post-natal period.

Post- partum or post-anal distress is the form of clinical illness that experienced during the postnatal period and can be classified into the following group of illness or diseases:

\section{a) Postpartum blues \\ b) postpartum psychosis \\ c) Postpartum depression.}

Most post-partum distress can start within the first month of delivery but can be delayed to start at the sixth month after delivery.
Postpartum depression is clinical symptom that manifest of feeling fatigue, Social withdrawal, Sadness, change in sleeping and eating pattern and guilt. Becoming mother could be difficult in seeing transformation from pregnant woman to mother in the childbearing age and this might not be familiar for lady that has new experience of being mother and responsible for new baby that require many responsibilities and daily task that should be done every day.

Multiple risk factors for postpartum distress have been suggested as no single cause has been identified. Personal vulnerability, personal trait and social factors such as un planned pregnancy, occupational instability, single parenthood and marital discord have been cited.

Mental disorders in general population is one of field recently receives good types of attention in term of diagnosis and management. This has mainly due to availability to some extent to mental health facilities that offering the mental services to population in countries with good health system that encompasses mental health as one of the key component of primary health care services .However in developing countries lack of mental health services is one of the main reasons behind steadily increase in reported cases.

Sudan is among the countries that yet have comprehensive mental facilities as limited numbers of facilities are identified and most of them are centralized in Khartoum, the capital of Sudan.

Allover Sudan there are only 17 PHC Mental Services scattered and two Mental Hospitals established in Khartoum, the capital of Sudan with total of 0.86 beds for 100,000 populations. None of these beds are reserved for children or Adolescents [7]. Professional counselling can be very beneficial and some health visitors offer counseling also important [8]. Perceived social support is strongly associated with enhanced life satisfaction and improved affect across the life span [9].

\section{Methodology}

\section{Study design}

Cross-sectional exploratory

\section{Study area}

South Darfur is second biggest city inhabitant of population after the capital Khartoum. 4,262,644 is the 
total population, out of them $1,027,297$ is women in the childbearing age.

The study was conducted in Nyala Teaching Hospital which located in Nyala, city that serve at least 2,000,000 population at the minimum and being as one of the main Hospital accepts and receives considerable numbers of referrals from different localities knowing the poor health setting in these areas. Study period: The study is exploratory so the timing is very limited and competing, three weeks is the duration of data collection from $8^{\text {th }}$ April-30th April, 2018.

\section{Population}

Postnatal mothers admitted to Nyala Teaching Hospital, South Darfur.

Sampling technique: Convenience sample targeting 60 women in the post-partum ward to be interviews through two types of questionnaire, the first is pertinent to demographic information and the second is assessment on Edinburgh post-partum mental scale.

Sampling is the 60 women in the postnatal period up to 6 weeks after delivery.

Inclusion criteria: Women in the post-partum in the selected Hospital at the time of data collection.

\section{Exclusion criteria}

$>$ Mothers who are having psychotic symptoms.

$>$ Mothers who are not willing to participate in study.

$>$ Mothers are not available at the time of study.

\section{Data Collection Technique}

The tool used for collection of the data is Edinburgh postnatal depression scale. The questionnaire has two parts, part 1 and part 2. Part 1 includes demographic variables such as age, sex, income, educational status and types of family.

These variables have been tried to be linked to the development of mental disorders and to which prevalence can be attributable for those who are proved by the below questionnaire in having mental distress.

Part 2 contain questionnaire on the post-natal distress which includes anxiety and depression. In this questionnaire 4 possible answers for each of 10 questions can be picked up by interviewed women and then the overall score categorizes women to which class they belong to. The classification either normal women in term of mental health or women suffering distress symptoms which can be diagnosed as depression or anxiety disorders. Women score above 13 meant that they are likely to have distress mental disorders which more likely depression and or anxiety.

\section{Results}

\begin{tabular}{|c|c|c|c|}
\hline Serial number & Demographic variables & Frequency & Percentage \\
\hline \multicolumn{4}{|c|}{ Age of mother in year } \\
\hline 1 & $<20$ years & 14 & $23.40 \%$ \\
\hline 2 & $21-25$ & 35 & $58.40 \%$ \\
\hline 4 & More than 30 & 1 & $1.60 \%$ \\
\hline \multicolumn{4}{|l|}{ Education } \\
\hline 1 & Primary & 18 & $30 \%$ \\
\hline 2 & Higher School & 34 & $56.60 \%$ \\
\hline 3 & Post-Graduates & 8 & $13.40 \%$ \\
\hline \multicolumn{4}{|l|}{ Occupation } \\
\hline 1 & House wives & 51 & $85 \%$ \\
\hline 2 & Daily wages & 4 & $6.60 \%$ \\
\hline 3 & Government Employee & 5 & $8.30 \%$ \\
\hline 4 & Private Sector Employee & 0 & 0 \\
\hline \multicolumn{4}{|l|}{ Types of family } \\
\hline 1 & Nuclear & 13 & $21.70 \%$ \\
\hline 2 & Expanded & 47 & $78 \% .3$ \\
\hline \multicolumn{4}{|l|}{ Number of children } \\
\hline 1 & One & 6 & $10 \%$ \\
\hline
\end{tabular}




\begin{tabular}{|c|c|c|c|}
\hline 2 & Two & 14 & $23.40 \%$ \\
\hline 3 & Three & 27 & $45 \%$ \\
\hline 4 & Four & 13 & 21.6 \\
\hline Child alive or dead & Alive & 54 & $90.00 \%$ \\
\hline 1 & Dead & 6 & $10 \%$ \\
\hline 2 & & & $46.70 \%$ \\
\hline Sex of child & Male & 28 & $53.30 \%$ \\
\hline 2 & Female & 32 & $75.60 \%$ \\
\hline Types of Delivery & Normal & 46 & $24.40 \%$ \\
\hline 1 & CS & 14 & 13.4 \\
\hline 2 & Accessible & 8 & 86.6 \\
\hline
\end{tabular}

Table 1: Demographic information.

The below table shows the demographic information that can reflect the prevalence of each in contributing to

post-partum mental distress.

\begin{tabular}{|c|c|c|c|}
\hline Total Number of women interviewed & Women with post-partum distress & & percentage \\
\hline 60 & Yes & 43 & $71.60 \%$ \\
\hline & No & 17 & $28.40 \%$ \\
\hline
\end{tabular}

Table 2: Results of Edinburgh Postnatal Depression Scale 1.

The below table is the result of 60 women assessed by Edinburgh postnatal depression scale to classify normal women from those who are having mental distress, anxiety and depression during post-natal period

\section{Discussion}

The current data in table 1 has considered all the demographic information: Age of mother, her education, occupation, type of family whether nuclear or expanded, number of children of children and type of delivery either normal or CS delivery.

It's apparent from the results the majority of interviewed women lie in the age group (21-25 years), 35 women in number at $58.4 \%$. Higher school education women have shown predominant of 34 women $(56.6 \%)$ compared to primary and postgraduates at $18(30 \%)$ and $8(13.4) \%$. This result concluded post- mental distress is more occurring in secondary school level compared to other education level for the studies women in this research this may contribute in appearance of problem and agree with that mentioned by Sheena et al, 2017.

51 women ( $85 \%$ ) out of the total 60 are house wives followed by government employee at $8.3 \%$ and daily labour at $6.6 \%$ these results are consistence with results obtained by Mohammadi (2011) in a research showed that depression degree of working women is less than housekeeping women. It's clear that huge responsibilities of the daily taking care of children and other home activities, and tasks have direct relations in developing post-partum distress and depression. This necessitate recommendation in increasing awareness among men to share some of responsibilities and support women by appreciation and motivation to overcome this kind of stress this not agree with Mohammadi (2011) because the studies were done in differ setting and population with differ beliefs .

Expanded family has override nuclear family with $78.3 \%$ compared to only $21.7 \%$. This might add additional work and stress on women in not taking care only of her children and husband but this might be extended to include parents and other close relatives. Its strongly have direct implication at the end for women to developed depression and other kind of stress during the postpartum period Siedlecki et al. Showed that social support causes one to overcome problems of marital life [10].

Pertinent to outcome of delivery, the majority of women delivered normal alive children with only 6 died. 
This result can show no correlation of postpartum distress and the outcome of delivery.

Neither sex of the child nor the outcome of delivery has implication on post-partum depression as 32 girls are delivered against 28 boys. In most Sudanese cultures, there is gender bias in favor of men who are dominating and looked at as future leaders. 46 women are delivered normally in comparison to 14 by caesarian section.

Pertinent to access to mental health services, most women $43(71.6 \%)$ have confirmed no accessibility to mental health services in case they are willing to seek mental services as there is only one psychiatric department within Nyala Teaching Hospital with limited mental services with only one Psychiatrist present in Nyala from time to time and this has negatively affected the mental services continuity.

\section{Conclusion}

The following conclusion has been drawn keeping in mind the finding of the present study.

The Post-partum mental distress have steadily increase during the recent years due to many reasons, bulk of them are due to non-availability of mental services to those seeking mental services. The prevalence is greater in developing countries with many factors incriminated in poor infrastructures and lack of awareness among communities on the importance of seeking such services for those are in need.

Competing factors have been the root cause in women presenting with mental distress during the postpartum period of which the overburden on women in taking care of expanded families, the poor education and the low income for most of families which as well a major factor in developing post-partum distress.

\section{Recommendation}

- Awareness is the key element in improving the overall accessibility to mental health services and reducing the prevalence of the post-partum distress in women in this period. This is could be done through mental health campaigns.

> Mental Health services need to be addressed as one key component of Primary health care and being integrated with other component to ensure package of services are delivered to communities at locality level with only complicated cases to be refer to Hospitals.
> South Darfur states, SMoH is urgently required to offer attractive environment for Psychiatrist and psychologist to work and settle. Not to mentioned good infrastructural set up of mental health institutions to ensure excellent quality of services are provided to those in need.

> The study can be replicated among rural community areas.

> The study can be replicated among mothers of home delivery.

> The tool may be modified to include the other factors affecting depression status of postnatal mothers.

- A comparative study can be conducted to assess the knowledge of rural and urban post-natal mothers.

$>$ A large-scale study needs to be carried out to generalize the findings.

- Early screening, treatment of postpartum depression and provision of psychosocial support should be part of routine prenatal care and should be considered.

\section{References}

1. WHO-AIMS (2009) Report on Mental Health System in Sudan. WHO and Ministry of Health, Khartoum, Sudan 26(12): 1-26.

2. Gavin NI, Gaynes BN, Lohr KN, Meltzer-Brody S, Gartlehner G, et al. (2005) Perinatal depression: a systematic review of prevalence and incidence. Obstet Gynecol 106(5): 1071-1083.

3. Pealstein T, Howard M, Salisbury A, Zlotnick C (2009) Postpartum depression. Am J Obstet Gynecol 200(4): 357-364.

4. Halbreich U, Karkun S (2006) Cross-cultural and social diversity of prevalenceofpostpartum depression and depressive symptoms. J Affect Disord 91(2-3): 97-111.

5. Kumar SV, Oliffe JL, Kelly MT (2018) Promoting Postpartum Mental Health in Fathers: Recommendations for Nurse Practitioners Am J Mens Health 12(2): 221-228.

6. Tukaram BZ, Prasanna D (2015) A Study to Assess the Level of Postnatal Depression among Postnatal Mothers Admitted in the Krishna Hospital, India, International Journal of Science and Research.

7. Zeinat BS, Murthy S, Khalid S (2009) Mental Health System in Sudan, Sudan, WHO, State Ministry of Health. 
8. Tukaram BZ, Prasanna D (2013) A Study to Assess the Level of Postnatal Depression among Postnatal Mothers Admitted in the Krishna Hospital, Karad, Int J Sci Res 13.

9. Siedlecki KL, Salthouse TA, Oishi S, Jeswani S (2014) The relationship between social support and subjective well-being across age. Social Indicators Research 117(2): 561-576.
10. Mohammadi A, Aghdam GA, Ranjic S (2011) Comparison of Postpartum Depression of Working Women and Housewives and its Relationship with Social Support and Marital Adjustment. Procedia Social and Behavioral Sciences 30: 1837-1839.

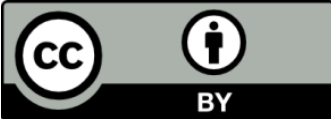

